\title{
On convexity properties of homogeneous functions of degree one*
}

\author{
Bernard Dacorogna \\ Département de Mathématiques, Ecole Polytechnique Fédérale de \\ Lausanne, 1015 Lausanne, Switzerland \\ Jean-Pierre Haeberly \\ Department of Mathematics, Fordham University, Bronx, NY 10458-5165, \\ U.S.A.
}

(MS received 15 February 1995. Revised MS received 25 May 1995)

\begin{abstract}
We provide an explicit example of a function that is homogeneous of degree one, rank-one convex, but not convex.
\end{abstract}

\section{Introduction}

Let $\mathbf{R}^{2 \times 2}$ denote the set of $2 \times 2$ real matrices and let $f: \mathbf{R}^{2 \times 2} \rightarrow \mathbf{R}$ be a continuous function that is homogeneous of degree one, i.e. it satisfies the following condition

$$
f(t \xi)=t f(\xi), \quad \text { for every } t \geqq 0 \text { and } \xi \in \mathbf{R}^{2 \times 2} \text {. }
$$

We would like to discuss the convexity properties of such functions. In addition to the usual notion of convexity, we need the following definition:

DEFINITION 1.1. $f: \mathbf{R}^{2 \times 2} \rightarrow \mathbf{R}$ is rank-one convex if

$$
f(t \xi+(1-t) \eta) \leqq t f(\xi)+(1-t) f(\eta)
$$

for every $t \in[0,1], \xi, \eta \in \mathbf{R}^{2 \times 2}$ with $\operatorname{det}(\xi-\eta)=0$ (where det stands for the determinant of the matrix).

Obviously any convex function is rank-one convex, while there are rank-one convex functions (such as $f(\xi)=\operatorname{det} \xi$ ) which are not convex. Surprisingly, if one imposes condition (1.1), then it is not clear that the two notions are not equivalent.

The first person to produce a counterexample was Müller [4], but in a very indirect way. In fact his result gives more than this (see below). Dacorogna [2] then showed that if, in addition to (1.1), $f$ is assumed to be rotationally invariant (in particular if $f(\xi)=g(|\xi|$, det $\xi)$, where $|\xi|$ denotes the Euclidean norm of the matrix, i.e. $|\xi|^{2}=\Sigma_{i, j=1}^{2} \xi_{i j}^{2}$ ), then any rank-one convex function is necessarily convex. Thus it remained an open question to find an explicit example of a function that is homogeneous of degree one and rank-one convex, but not convex. We produce here a family of such examples. Before describing our results, we should emphasise that

* This research was supported in part by a grant from the Fonds National Suisse pour la Recherche Scientifique. 
these functions and notions are important in the Calculus of Variations (see [1]). There, the notion of quasiconvexity plays the central role. It is well known that convexity implies quasiconvexity and quasiconvexity implies rank-one convexity. Müller's example gives in fact an example of a quasiconvex function that is not convex. It is not presently known whether our examples are quasiconvex.

We now introduce some notation. It will be more convenient to identify $\mathbf{R}^{2 \times 2}$ with $\mathbf{R}^{4}$ and, therefore, a matrix $\xi$ will be written as a vector $\left(\xi_{1}, \xi_{2}, \xi_{3}, \xi_{4}\right)$. We then let

$$
\left\{\begin{array}{l}
\langle\xi ; \eta\rangle=\sum_{i=1}^{4} \xi_{i} \eta_{i}, \quad|\xi|^{2}=\langle\xi ; \xi\rangle, \\
\tilde{\xi}=\left(\xi_{4},-\xi_{3},-\xi_{2}, \xi_{1}\right), \\
\operatorname{det} \xi=\xi_{1} \xi_{4}-\xi_{2} \xi_{3}=\frac{1}{2}\langle\xi ; \xi\rangle .
\end{array}\right.
$$

Note that $\tilde{\xi}$ is just the gradient of det $\xi$. Consider the matrix $E \in \mathbf{R}^{4 \times 4}$ representing the quadratic form det. It is defined as

$$
E=\left(\begin{array}{rrrr}
0 & 0 & 0 & 1 \\
0 & 0 & -1 & 0 \\
0 & -1 & 0 & 0 \\
1 & 0 & 0 & 0
\end{array}\right)
$$

Then

$$
E \xi=\tilde{\xi} \text { and } \operatorname{det} \xi=\frac{1}{2}\langle E \xi ; \xi\rangle .
$$

Finally, our counterexample will be of the form

$$
f(\xi)= \begin{cases}|\xi|+\gamma \frac{\langle M \xi ; \xi\rangle}{|\xi|} & \text { if } \xi \neq 0, \\ 0 & \text { if } \xi=0,\end{cases}
$$

where $\gamma \geqq 0$ and $M \in \mathbf{R}^{4 \times 4}$ is a symmetric matrix whose eigenvalues are $\mu_{1} \leqq \mu_{2} \leqq \mu_{3} \leqq \mu_{4}$.

We will see in the following theorems that choosing $M$ and $\gamma$ appropriately will produce rank-one convex functions, $f$, which are not convex.

THEOREM 1.2. Let $f, M$ and $\gamma$ be as above. Then

$$
f \text { is convex } \Leftrightarrow \gamma \leqq \gamma_{c} \text {, }
$$

where

$$
\gamma_{c}= \begin{cases}\frac{1}{\mu_{4}-2 \mu_{1}} & \text { if } \mu_{4}-2 \mu_{1}>0, \\ +\infty & \text { if } \mu_{4}-2 \mu_{1} \leqq 0 .\end{cases}
$$

REMARK 1.3. It will be obvious from the proof that if $\gamma \leqq 0$, then $f$ is convex if and only if

$$
-\gamma \leqq \frac{1}{2 \mu_{4}-\mu_{1}}
$$


THEOREM 1.4. Let $f, M$ and $\gamma$ be as above, and let $\varphi_{i}, 1 \leqq i \leqq 4$, denote an orthonormal set of eigenvectors corresponding to the $\mu_{i}$ 's. Assume further that $M$ commutes with $E$. Then two cases can happen:

CASE 1. If $\operatorname{det} \varphi_{4}=-\operatorname{det} \varphi_{1}$, then

$$
f \text { is rank-one convex } \Leftrightarrow f \text { is convex. }
$$

CASE 2. If $\operatorname{det} \varphi_{4}=\operatorname{det} \varphi_{1}$, then

$$
f \text { is rank-one convex } \Leftrightarrow \gamma \leqq \gamma_{r},
$$

where

$$
\gamma_{r}= \begin{cases}\frac{1}{\gamma_{2}}\left\{\frac{1}{\gamma_{1}}, \frac{1}{\gamma_{2}}\right\} & \text { if } \gamma_{1}>0 \text { and } \gamma_{2}>0, \\ \frac{1}{\gamma_{1}} & \text { if } \gamma_{1} \leqq 0 \text { and } \gamma_{2}>0, \\ +\infty & \text { if } \gamma_{1}>0 \text { and } \gamma_{2} \leqq 0, \\ \text { if } \gamma_{1} \leqq 0 \text { and } \gamma_{2} \leqq 0\end{cases}
$$

and

$$
\gamma_{1}=\frac{\mu_{4}+\mu_{3}}{2}-2 \mu_{1}, \quad \gamma_{2}=\mu_{4}-\left(\mu_{1}+\mu_{2}\right)
$$

The following corollary is an immediate consequence of the two theorems.

COROllary 1.5. Consider the function

$$
g(\xi)=\frac{\langle M \xi ; \xi\rangle}{|\xi|}
$$

(1) Let $M$ be as in Theorem 1.2. Then

$$
g \text { is convex } \Leftrightarrow 2 \mu_{1}-\mu_{4} \geqq 0 .
$$

(2) Let $M$ be as in Theorem 1.4 with $\operatorname{det} \varphi_{1}=\operatorname{det} \varphi_{4}$. (If $\operatorname{det} \varphi_{1}=-\operatorname{det} \varphi_{4}$, see the convex case.) Then

$$
g \text { is rank-one convex } \Leftrightarrow\left\{\begin{array}{c}
\mu_{1}+\mu_{2}-\mu_{4} \geqq 0 \\
\text { and } \\
2 \mu_{1}-\frac{\mu_{3}+\mu_{4}}{2} \geqq 0 .
\end{array}\right.
$$

REMARK 1.6. It is interesting to compare the corollary with the case of quadratic forms. It is well known that the function

$$
q(\xi)=\langle M \xi ; \xi\rangle \text { is convex } \Leftrightarrow \mu_{1} \geqq 0 .
$$

Under the hypotheses of Theorem 1.4, one can show by a similar but simpler 
argument that

$$
q \text { is rank-one convex } \Leftrightarrow \begin{cases}\frac{\mu_{1}+\mu_{2}}{2} \geqq 0 & \text { if } \operatorname{det} \varphi_{1}=-\operatorname{det} \varphi_{2}, \\ \frac{\mu_{1}+\mu_{3}}{2} \geqq 0 & \text { if } \operatorname{det} \varphi_{1}=\operatorname{det} \varphi_{2} .\end{cases}
$$

RemarK 1.7. Similar results as in Theorem 1.4 can be derived for $\gamma<0$. More precisely,

$$
f \text { is rank-one convex } \Leftrightarrow\left\{\begin{array}{l}
1-|\gamma|\left(2 \mu_{4}-\frac{\mu_{1}+\mu_{2}}{2}\right) \geqq 0 \\
\text { and } \\
1-|\gamma|\left(\mu_{4}+\mu_{3}-\mu_{1}\right) \geqq 0 .
\end{array}\right.
$$

Remark 1.8. The fact that in Theorem 1.4 we have to add the extra hypothesis on $M$ just means that $\gamma_{r}$ depends not only on the eigenvalues of $M$, but also on the eigenvectors, while $\gamma_{c}$ depends only on the eigenvalues.

REMARK 1.9. With the help of the theorems we may now give an explicit example. Let

$$
M=\left(\begin{array}{llll}
9 & 0 & 0 & 1 \\
0 & 6 & 2 & 0 \\
0 & 2 & 6 & 0 \\
1 & 0 & 0 & 9
\end{array}\right)
$$

its eigenvalues are $\mu_{1}=4 \leqq \mu_{2}=\mu_{3}=8 \leqq \mu_{4}=10$, with eigenvectors

$$
\varphi_{1}=\frac{1}{\sqrt{2}}(0,1,-1,0), \quad \varphi_{2}=\frac{1}{\sqrt{2}}(0,1,1,0), \quad \varphi_{3}=\frac{1}{\sqrt{2}}(1,0,0,-1) .
$$

and

$$
\varphi_{4}=\frac{1}{\sqrt{2}}(1,0,0,1)
$$

It commutes with $E$ and

$$
\gamma_{c}=\frac{1}{2}, \quad \gamma_{r}=1
$$

Therefore, choosing $\gamma \in\left(\frac{1}{2}, 1\right]$ gives the explicit counterexample.

REMARK 1.10. Theorems 1.2 and 1.4 should be compared with [2] when $f$ is rotationally invariant. In particular, if $M=E$, i.e.

$$
f(\xi)= \begin{cases}|\xi|+2 \gamma \frac{\operatorname{det} \xi}{|\xi|} & \text { if } \xi \neq 0, \\ 0 & \text { if } \xi=0\end{cases}
$$


we find (as in [2]) that

$$
\gamma_{c}=\gamma_{r}=\frac{1}{3}
$$

since the eigenvalues are $\mu_{1}=\mu_{2}=-1$ and $\mu_{3}=\mu_{4}=1$.

REMARK 1.11. Of course our results do not settle the quasiconvexity of $f$. It is easy to see that $f$ is quasiconvex if and only if $\gamma \leqq \gamma_{q}$ for a certain $\gamma_{q}$. By the general fact, we have $\gamma_{c} \leqq \gamma_{q} \leqq \gamma_{r}$. The question is to decide whether $\gamma_{q}=\gamma_{r}$ or $\gamma_{q}<\gamma_{r}$. If equality holds, then we would have an explicit example of a quasiconvex function satisfying (1.1) which is not convex (as in [4]). Obviously, the second possibility would be much more interesting and would settle the long-standing question of the equivalence of quasiconvexity and rank-one convexity (see [5] for a counterexample in higher dimensions). Our numerical results, presented in a forthcoming paper [3], tend to show that $\gamma_{q}=\gamma_{r}$.

\section{Proof of Theorem 1.2}

We start by computing the Hessian of $f$ at $\xi \neq 0$. Observe first that

$$
\begin{aligned}
\frac{\partial f(\xi)}{\partial \xi_{\alpha}} & =\frac{\xi_{\alpha}}{|\xi|}+\gamma \frac{2|\xi|(M \xi)_{\alpha}-\langle M \xi ; \xi\rangle \frac{\xi_{\alpha}}{|\xi|}}{|\xi|^{2}} \\
& =\frac{\xi_{\alpha}}{|\xi|}+\gamma \frac{2|\xi|^{2}(M \xi)_{\alpha}-\langle M \xi ; \xi\rangle \xi_{\alpha}}{|\xi|^{3}}
\end{aligned}
$$

We then have

$$
\begin{aligned}
\frac{\partial^{2} f(\xi)}{\partial \xi_{\alpha} \partial \xi_{\beta}}= & \frac{\delta_{\alpha \beta}}{|\xi|}-\frac{\xi_{\alpha} \xi_{\beta}}{|\xi|^{3}} \\
& +\frac{\gamma}{|\xi|^{6}}\left\{\left[4(M \xi)_{\alpha} \xi_{\beta}+2|\xi|^{2} M_{\alpha \beta}-\langle M \xi ; \xi\rangle \delta_{\alpha \beta}-2(M \xi)_{\beta} \xi_{\alpha}\right]|\xi|^{3}\right. \\
& \left.-3|\xi| \xi_{\beta}\left[2|\xi|^{2}(M \xi)_{\alpha}-\langle M \xi ; \xi\rangle \xi_{\alpha}\right]\right\},
\end{aligned}
$$

where $\delta_{\alpha \beta}$ is the Kronecker symbol. Thus, we get that, for any $\xi \neq 0$,

$$
\begin{aligned}
\sum_{\alpha, \beta=1}^{4} \frac{\partial^{2} f(\xi)}{\partial \xi_{\alpha} \partial \xi_{\beta}} \lambda_{\alpha} \lambda_{\beta}= & \frac{|\lambda|^{2}}{|\xi|}-\frac{(\langle\xi ; \lambda\rangle)^{2}}{|\xi|^{3}} \\
& +\frac{\gamma}{|\xi|^{5}}\left\{|\xi|^{2}\left[2\langle M \xi ; \lambda\rangle\langle\xi ; \lambda\rangle+2|\xi|^{2}\langle M \lambda ; \lambda\rangle-\langle M \xi ; \xi\rangle|\lambda|^{2}\right]\right. \\
& \left.-3\langle\xi ; \lambda\rangle\left[2|\xi|^{2}\langle M \xi ; \lambda\rangle-\langle M \xi ; \xi\rangle\langle\xi ; \lambda\rangle\right]\right\} \\
= & \frac{|\lambda|^{2}}{|\xi|}-\frac{(\langle\xi ; \lambda\rangle)^{2}}{|\xi|^{3}} \\
& +\frac{\gamma}{|\xi|^{5}}\left\{2|\xi|^{4}\langle M \lambda ; \lambda\rangle-4|\xi|^{2}\langle M \xi ; \lambda\rangle\langle\xi ; \lambda\rangle\right. \\
& \left.-|\xi|^{2}|\lambda|^{2}\langle M \xi ; \xi\rangle+3(\langle\xi ; \lambda\rangle)^{2}\langle M \xi ; \xi\rangle\right\}
\end{aligned}
$$


It is clear that $f$ will be convex if and only if the quadratic form in (2.1) is positive for every $\xi \neq 0$ and $\lambda$. The fact that $f$ is not differentiable at 0 does not cause any trouble in this case. Therefore

$$
f \text { is convex } \Leftrightarrow \inf _{\xi \neq 0} \inf _{\lambda}\left\{\sum_{\alpha, \beta=1}^{4} \frac{\partial^{2} f(\xi)}{\partial \xi_{\alpha} \partial \xi_{\beta}} \lambda_{\alpha} \lambda_{\beta}=\left\langle\nabla^{2} f(\xi) \lambda ; \lambda\right\rangle\right\} \geqq 0 .
$$

Since the quadratic form is homogeneous of degree -1 in $\xi$, we may assume that $|\xi|=1$. We may also write

$$
\lambda=t \xi+s \eta, \quad \text { with } t, s \in \mathbf{R},|\eta|=1, \quad \text { and } \quad\langle\xi ; \eta\rangle=0 .
$$

We then obtain that

$$
\begin{gathered}
|\lambda|^{2}=t^{2}+s^{2}, \quad\langle\xi ; \lambda\rangle=t, \quad\langle M \xi, \lambda\rangle=t\langle M \xi ; \xi\rangle+s\langle M \xi ; \eta\rangle \\
\langle M \lambda ; \lambda\rangle=t^{2}\langle M \xi ; \xi\rangle+2 s t\langle M \xi ; \eta\rangle+s^{2}\langle M \eta ; \eta\rangle .
\end{gathered}
$$

So, coming back to the quadratic form, we have for $|\xi|=|\eta|=1$,

$$
\left\langle\nabla^{2} f(\xi) \lambda ; \lambda\right\rangle=s^{2}\{1+\gamma[2\langle M \eta ; \eta\rangle-\langle M \xi ; \xi\rangle]\} .
$$

We finally get that

$$
f \text { is convex } \Leftrightarrow \inf _{\substack{|\xi|=|\eta|=1 \\\langle\xi ; \eta\rangle=0}}\{1+\gamma[2\langle M \eta ; \eta\rangle-\langle M \xi ; \xi\rangle]\} \geqq 0 .
$$

Since $\gamma \geqq 0$, it is clear that the minimum is attained when $\langle M \eta ; \eta\rangle$ is minimum and $\langle M \xi ; \xi\rangle$ is maximum, i.e. when $\eta=\varphi_{1}$ (the eigenvector corresponding to the smallest eigenvalue $\mu_{1}$ ) and $\xi=\varphi_{4}$ (the eigenvector corresponding to the largest eigenvalue $\left.\mu_{4}\right)$. Thus

$$
f \text { is convex } \Leftrightarrow 1+\gamma\left(2 \mu_{1}-\mu_{4}\right) \geqq 0 .
$$

The conclusion of the theorem follows at once. One also notices that if $\gamma \leqq 0$, then the same argument leads to

$$
f \text { is convex } \Leftrightarrow 1+\gamma\left(2 \mu_{4}-\mu_{1}\right) \geqq 0 .
$$

\section{Proof of Theorem 1.4}

We divide the proof into three steps.

Step 1. It is clear that, even though $f$ is not differentiable at 0 , we have

$$
f \text { is rank-one convex } \Leftrightarrow \inf _{\xi \neq 0} \inf _{\operatorname{det} \lambda=0}\left\{\left\langle\nabla^{2} f(\xi) \lambda ; \lambda\right\rangle\right\} \geqq 0 .
$$

Writing $\lambda=t \xi+s \eta$ with $t, s \in \mathbf{R},\langle\xi ; \eta\rangle=0,|\xi|=|\eta|=1$, we find as in the proof of Theorem 1.2 that (see (2.2)) $f$ is a rank-one convex if and only if

$$
\begin{gathered}
\inf \{1+\gamma[2\langle M \eta ; \eta\rangle-\langle M \xi ; \xi\rangle]:|\xi|=|\eta|=1,\langle\xi ; \eta\rangle=\operatorname{det}(t \xi+s \eta)=0, \\
\left.t, s \in \mathbf{R} \text { with } t^{2}+s^{2} \neq 0\right\} \geqq 0 .
\end{gathered}
$$

Since $\gamma \geqq 0$, we finally deduce that if

$$
\begin{gathered}
m=\inf \{2\langle M \eta ; \eta\rangle-\langle M \xi ; \xi\rangle:|\xi|=|\eta|=1,\langle\xi ; \eta\rangle=\operatorname{det}(t \xi+s \eta)=0, \\
\left.t, s \in \mathbf{R} \text { with } t^{2}+s^{2} \neq 0\right\}
\end{gathered}
$$


then

$$
f \text { is rank-one convex } \Leftrightarrow 1+\gamma m \geqq 0 \text {. }
$$

(If $\gamma \leqq 0$, then one has to compute, analogously, the sup in (3.1).) We will show in the next steps that, in case 1 ,

$$
m=2 \mu_{1}-\mu_{4}
$$

while in case 2 ,

$$
m=\min \left\{\mu_{1}+\mu_{2}-\mu_{4}, 2 \mu_{1}-\frac{\mu_{3}+\mu_{4}}{2}\right\} .
$$

Combining (3.2), (3.3), and (3.4) will then give the claimed result.

Step 2 . Since $M$ commutes with $E$, we necessarily have

$$
\begin{gathered}
\tilde{\varphi}_{i}=E \varphi_{i}= \pm \varphi_{i}, \\
\operatorname{det} \varphi_{i}=\frac{1}{2}\left\langle\tilde{\varphi}_{i} ; \varphi_{i}\right\rangle= \pm \frac{1}{2},
\end{gathered}
$$

for $1 \leqq i \leqq 4$. Therefore, cases 1 and 2 do cover all possibilities, since two of the det $\varphi_{i}$ are $+\frac{1}{2}$ and two are $-\frac{1}{2}$.

We then immediately get the theorem in case 1 . Indeed, choose $\eta=\varphi_{1}, \xi=\varphi_{4}$, $s=t=1$, and observe that they are admissible for the minimisation in (3.1). This choice leads to

$$
m=2 \mu_{1}-\mu_{4}
$$

Hence, we get (cf. Theorem 1.2)

$$
f \text { is rank-one convex } \Rightarrow 1+\gamma\left(2 \mu_{1}-\mu_{4}\right) \geqq 0 \Rightarrow \gamma \leqq \gamma_{c} .
$$

Since, by Theorem 1.2, we have

$$
\gamma \leqq \gamma_{c} \Rightarrow f \text { is convex }
$$

and, trivially, $f$ convex implies that $f$ is rank-one convex, we have indeed established the theorem in case 1 .

Step 3. From now on, we will assume that $\operatorname{det} \varphi_{1}=\operatorname{det} \varphi_{4}$, so that the choice $\eta=\varphi_{1}$ and $\xi=\varphi_{4}$ is no longer admissible in (3.1) for any choice of $s, t \in \mathbf{R}$ with $s^{2}+t^{2} \neq 0$. We will prove that the right choice is either

$$
\eta=\frac{1}{\sqrt{2}}\left(\varphi_{1}+\varphi_{2}\right), \quad \xi=\varphi_{4}, \quad \text { and } \quad t=0, s=1,
$$

or

$$
\eta=\varphi_{1}, \quad \xi=\frac{1}{\sqrt{2}}\left(\varphi_{3}+\varphi_{4}\right), \quad \text { and } \quad t=1, s=0 .
$$

Let us write any $\xi, \eta \in \mathbf{R}^{4}$ as

$$
\xi=\sum_{i=1}^{4} \xi_{i} \varphi_{i}, \quad \eta=\sum_{i=1}^{4} \eta_{i} \varphi_{i}
$$


and observe that

$$
2\langle M \eta ; \eta\rangle-\langle M \xi ; \xi\rangle=\sum_{i=1}^{4} \mu_{i}\left(2 \eta_{i}^{2}-\xi_{i}^{2}\right)
$$

If (3.4) holds, this means that for every $\xi, \eta \in \mathbf{R}^{4}, s, t \in \mathbf{R}$ with $s^{2}+t^{2} \neq 0$ such that

$$
\left\{\begin{array}{c}
|\xi|^{2}=\xi_{1}^{2}+\xi_{2}^{2}+\xi_{3}^{2}+\xi_{4}^{2}=|\eta|^{2}=\eta_{1}^{2}+\eta_{2}^{2}+\eta_{3}^{2}+\eta_{4}^{2}=1 \\
\langle\xi ; \eta\rangle=0 \Leftrightarrow \xi_{1} \eta_{1}+\xi_{4} \eta_{4}=-\left(\xi_{2} \eta_{2}+\xi_{3} \eta_{3}\right) \\
\operatorname{det}(t \xi+s \eta)=0 \Leftrightarrow t^{2}\left(\xi_{1}^{2}+\xi_{4}^{2}-\xi_{2}^{2}-\xi_{3}^{2}\right)+s^{2}\left(\eta_{1}^{2}+\eta_{4}^{2}-\eta_{2}^{2}-\eta_{3}^{2}\right) \\
+2 t s\left(\xi_{1} \eta_{1}+\xi_{4} \eta_{4}-\xi_{2} \eta_{2}-\xi_{3} \eta_{3}\right)=0
\end{array}\right.
$$

we have to prove that

$$
\left\{\begin{array}{l}
\sum_{i=1}^{4} \mu_{i}\left(2 \eta_{i}^{2}-\xi_{i}^{2}\right) \geqq \mu_{1}+\mu_{2}-\mu_{4} \\
\quad \text { or } \\
\sum_{i=1}^{4} \mu_{i}\left(2 \eta_{i}^{2}-\xi_{i}^{2}\right) \geqq 2 \mu_{1}-\frac{\mu_{3}+\mu_{4}}{2} .
\end{array}\right.
$$

So we now have to show that (3.6) holds whenever (3.5) does. We will transform (3.6) into more amenable inequalities. Using the facts that $|\xi|=|\eta|=1$, we get that (3.6) is equivalent to

$$
\left\{\begin{array}{l}
\mu_{1}\left(\eta_{1}^{2}-\eta_{2}^{2}-\eta_{3}^{2}-\eta_{4}^{2}-\xi_{1}^{2}\right)+\mu_{2}\left(\eta_{2}^{2}-\eta_{1}^{2}-\eta_{3}^{2}-\eta_{4}^{2}-\xi_{2}^{2}\right)+\mu_{3}\left(2 \eta_{3}^{2}-\xi_{3}^{2}\right) \\
\quad+\mu_{4}\left(2 \eta_{4}^{2}+\xi_{1}^{2}+\xi_{2}^{2}+\xi_{3}^{2}\right) \geqq 0 \\
\quad \text { or } \\
\mu_{1}\left(-2 \eta_{2}^{2}-2 \eta_{3}^{2}-2 \eta_{4}^{2}-\xi_{1}^{2}\right)+\mu_{2}\left(2 \eta_{2}^{2}-\xi_{2}^{2}\right)+\mu_{3}\left(2 \eta_{3}^{2}-\frac{1}{2} \xi_{3}^{2}+\frac{1}{2} \xi_{2}^{2}+\frac{1}{2} \xi_{1}^{2}+\frac{1}{2} \xi_{4}^{2}\right) \\
\quad+\mu_{4}\left(2 \eta_{4}^{2}-\frac{1}{2} \xi_{4}^{2}+\frac{1}{2} \xi_{1}^{2}+\frac{1}{2} \xi_{2}^{2}+\frac{1}{2} \xi_{3}^{2}\right) \geqq 0 .
\end{array}\right.
$$

Rewriting the above inequalities, we find that

$$
\left\{\begin{array}{l}
\left(\mu_{2}-\mu_{1}\right)\left(\eta_{2}^{2}-\eta_{1}^{2}\right)+\left(2 \mu_{3}-\mu_{1}-\mu_{2}\right) \eta_{3}^{2}+\left(2 \mu_{4}-\mu_{1}-\mu_{2}\right) \eta_{4}^{2} \\
\quad+\left(\mu_{4}-\mu_{1}\right) \xi_{1}^{2}+\left(\mu_{4}-\mu_{2}\right) \xi_{2}^{2}+\left(\mu_{4}-\mu_{3}\right) \xi_{3}^{2} \geqq 0 \\
\quad \text { or } \\
2\left(\mu_{2}-\mu_{1}\right) \eta_{2}^{2}+2\left(\mu_{3}-\mu_{1}\right) \eta_{3}^{2}+2\left(\mu_{4}-\mu_{1}\right) \eta_{4}^{2}+\left(\frac{\mu_{4}+\mu_{3}}{2}-\mu_{1}\right) \xi_{1}^{2} \\
+\left(\frac{\mu_{4}+\mu_{3}}{2}-\mu_{2}\right) \xi_{2}^{2}+\frac{1}{2}\left(\mu_{4}-\mu_{3}\right)\left(\xi_{3}^{2}-\xi_{4}^{2}\right) \geqq 0
\end{array}\right.
$$

Since $\mu_{4} \geqq \mu_{3} \geqq \mu_{2} \geqq \mu_{1}$, we find that if $\mu_{2}=\mu_{1}$ or $\mu_{4}=\mu_{3}$, then one of the above inequalities is satisfied. So we may assume that $\mu_{3} \neq \mu_{4}$ and $\mu_{1} \neq \mu_{2}$.

We transform the inequalities again and get the following formulation, still 
equivalent to $(3.6)$ :

$$
\left\{\begin{array}{l}
\eta_{2}^{2}+\eta_{3}^{2}+\eta_{4}^{2}-\eta_{1}^{2}+\xi_{1}^{2}+\frac{\mu_{4}-\mu_{2}}{\mu_{2}-\mu_{1}}\left(2 \eta_{4}^{2}+\xi_{1}^{2}+\xi_{2}^{2}\right)+2 \frac{\mu_{3}-\mu_{2}}{\mu_{2}-\mu_{1}} \eta_{3}^{2}+\frac{\mu_{4}-\mu_{3}}{\mu_{2}-\mu_{1}} \xi_{3}^{2} \geqq 0, \\
\text { or } \\
\frac{1}{2}\left(\xi_{1}^{2}+\xi_{2}^{2}+\xi_{3}^{2}-\xi_{4}^{2}\right)+2 \eta_{4}^{2}+\frac{\mu_{3}-\mu_{1}}{\mu_{4}-\mu_{3}}\left(2 \eta_{3}^{2}+2 \eta_{4}^{2}+\xi_{1}^{2}\right)+2 \frac{\mu_{2}-\mu_{1}}{\mu_{4}-\mu_{3}} \eta_{2}^{2}+\frac{\mu_{3}-\mu_{2}}{\mu_{4}-\mu_{3}} \xi_{2}^{2} \geqq 0 .
\end{array}\right.
$$

From now on, we proceed by contradiction and assume that there exist $\xi, \eta, s, t$ as in (3.5) but that (3.6) does not hold. This means, using (3.7), that, in addition to (3.5), $\xi$ and $\eta$ satisfy

$$
\left\{\begin{aligned}
& \eta_{1}^{2}-\eta_{2}^{2}-\eta_{3}^{2}>\eta_{4}^{2}+\xi_{1}^{2}+\frac{\mu_{4}-\mu_{2}}{\mu_{2}-\mu_{1}}\left(2 \eta_{4}^{2}+\xi_{1}^{2}+\xi_{2}^{2}\right)+2 \frac{\mu_{3}-\mu_{2}}{\mu_{2}-\mu_{1}} \eta_{3}^{2}+\frac{\mu_{4}-\mu_{3}}{\mu_{2}-\mu_{1}} \xi_{3}^{2} \\
& \geqq \frac{\left(\mu_{4}-\mu_{2}\right) \xi_{2}^{2}+\left(\mu_{4}-\mu_{3}\right) \xi_{3}^{2}}{\mu_{2}-\mu_{1}} \\
& \geqq \frac{\mu_{4}-\mu_{3}}{\mu_{2}-\mu_{1}}\left(\xi_{2}^{2}+\xi_{3}^{2}\right) \\
& \text { and } \quad \\
& \xi_{4}^{2}-\xi_{2}^{2}-\xi_{3}^{2}>\xi_{1}^{2}+4 \eta_{4}^{2}+2 \frac{\mu_{3}-\mu_{1}}{\mu_{4}-\mu_{3}}\left(2 \eta_{3}^{2}+2 \eta_{4}^{2}+\xi_{1}^{2}\right)+4 \frac{\mu_{2}-\mu_{1}}{\mu_{4}-\mu_{3}} \eta_{2}^{2}+2 \frac{\mu_{3}-\mu_{2}}{\mu_{4}-\mu_{3}} \xi_{2}^{2} \\
& \geqq 4 \frac{\left(\mu_{3}-\mu_{1}\right) \eta_{3}^{2}+\left(\mu_{2}-\mu_{1}\right) \eta_{2}^{2}}{\mu_{4}-\mu_{3}} \\
& \geqq 4 \frac{\mu_{2}-\mu_{1}}{\mu_{4}-\mu_{3}}\left(\eta_{2}^{2}+\eta_{3}^{2}\right) .
\end{aligned}\right.
$$

We now use the facts that $\operatorname{det}(t \xi+s \eta)=0,\langle\xi ; \eta\rangle=0$, and the strict inequalities above, to get

$$
\begin{aligned}
0 & =t^{2}\left(\xi_{1}^{2}+\xi_{4}^{2}-\xi_{2}^{2}-\xi_{3}^{2}\right)+s^{2}\left(\eta_{1}^{2}+\eta_{4}^{2}-\eta_{2}^{2}-\eta_{3}^{2}\right)-4 t s\left(\xi_{2} \eta_{2}+\xi_{3} \eta_{3}\right) \\
& >t^{2}\left[\xi_{1}^{2}+4 \frac{\mu_{2}-\mu_{1}}{\mu_{4}-\mu_{3}}\left(\eta_{2}^{2}+\eta_{3}^{2}\right)\right]+s^{2}\left[\eta_{4}^{2}+\frac{\mu_{4}-\mu_{3}}{\mu_{2}-\mu_{1}}\left(\xi_{2}^{2}+\xi_{3}^{2}\right)\right]-4|t s|\left|\xi_{2} \eta_{2}+\xi_{3} \eta_{3}\right| \\
& \geqq \frac{\mu_{4}-\mu_{3}}{\mu_{2}-\mu_{1}} s^{2}\left(\xi_{2}^{2}+\xi_{3}^{2}\right)-4|t s|\left|\xi_{2} \eta_{2}+\xi_{3} \eta_{3}\right|+4 \frac{\mu_{2}-\mu_{1}}{\mu_{4}-\mu_{3}} t^{2}\left(\eta_{2}^{2}+\eta_{3}^{2}\right)
\end{aligned}
$$

Hence, using the Cauchy-Schwarz inequality on the middle term, we get

$$
0>\left[\left(\mu_{4}-\mu_{3}\right)|s| \sqrt{\xi_{2}^{2}+\xi_{3}^{2}}-2\left(\mu_{2}-\mu_{1}\right)|t| \sqrt{\eta_{2}^{2}+\eta_{3}^{2}}\right]^{2}
$$

which is absurd. Therefore, if (3.5) holds, then (3.6) does also. This concludes the proof of the theorem. 


\section{References}

1 B. Dacorogna. Direct Methods in the Calculus of Variations (Berlin: Springer, 1989).

2 B. Dacorogna. On rank one convex functions which are homogeneous of degree one. In Progress in partial differential equations, Pont-à-Mousson, 1994, Vol. 2, eds C. Bandle et al. (Harlow: Longman, 1995).

3 B. Dacorogna and J.-P. Haeberly. Some numerical methods for the study of the convexity notions arising in the calculus of variations (in prep.).

4 S. Müller. On quasiconvex functions which are homogeneous of degree one. Indiana Univ. Math. J. 41 (1992), 295-300.

5 V. Sverak. Rank one convexity does not imply quasiconvexity. Proc. Roy. Soc. Edinburgh Sect. A 120 (1992), 185-9.

(Issued 14 October 1996) 